\title{
CORONARY HEART DISEASE COLLABORATIVE: LEARNING FROM PATIENT AND CARER EXPERIENCE
}

\author{
Tricia Myers, Programme Manager \\ Lancashire and South Cumbria CHD Collaborative
}

This article is intended to give an overview of the work of the Coronary Heart Disease Collaborative (CHDC) generally and the use of discovery interviews in particular. The National Service Framework (NSF) for Coronary Heart Disease (CHD) published in March 2000 is a ten year programme ${ }^{(1)}$. The NSF set standards to be achieved and the framework for action to:

- prevent disease, save more lives and improve quality of life

- deliver services that are responsive to the needs and choices of patients

- reduce inequalities, through action to reduce the risk of CHD.

All patients should have access to prompt, high-quality care across the patient pathway, as set out in the NSF.

Excellent progress has been made in the first four years in modernising cardiac services and reducing variation in access to services across the country but there is still a long way to go.

The CHDC is a key element in the delivery of the standards set out in the CHD NSF. It is one of the largest modernisation programmes within the NHS Modernisation Agency, starting work in October 2000 with 10 pilot sites; it now involves 30 local CHDC programmes covering every strategic health authority in England.

The collaborative approach is to get managers, clinicians and the whole multidisciplinary team to work together and across organisations to review the NSF standards and progress being made towards achieving them. The CHDC areas of work mirror the chapters of the NSF, excluding primary prevention. These are: secondary prevention, heart failure and palliative care; angina and interventional cardiology; acute myocardial infarction and acute coronary syndrome; cardiac surgery; and cardiac rehabilitation. Since 2001, hundreds of service improvements have been made using tools such as demand and capacity analysis, statistical process control and process mapping and more recently discovery interviews ${ }^{(2)}$.

These service improvements are being spread widely across primary, secondary and tertiary services as well as ambulance trusts, while new ways of delivering better services are being continually tested and implemented.

\section{CHDC goal}

"Our goal is to improve the experience and outcomes for people who have, or are at risk of developing, heart disease by redesigning the whole pathway of care."
Our goal will remain patient- and carer-focussed, with emphasis on supporting the delivery of the Priorities and Planning Framework (PPF) ${ }^{(3)}$ targets and NSF standards by working with local communities to streamline the patient pathway and improve patient experience. The collaborative is not unique in helping to deliver new solutions to service delivery. The intention is that by integrating the innovatory redesign work into the delivery of local cardiac network strategies to improve services, health communities will be enabled to work continuously with this improvement approach. Cooperation with other redesign programmes such as critical care and primary care is actively pursued.

Now based in Preston Business Centre with the Cardiac Network, the Lancashire and South Cumbria CHD Collaborative Programme Team was established in June 2002, initially working in East Lancashire. It can usefully be described as the innovation arm of the Cardiac Network, supporting modernisation and helping implementation of local delivery plans.

It has recently commenced work across the Cardiac Network area, including Morecambe Bay. Sally Thornton, project manager, commenced working on service redesign issues across the bay for the CHDC in December 2003. Initially her work has been in establishing contact with key people in cardiac services, promoting the work of the CHDC and planning review events with local staff.

As mentioned above, the programme uses a number of tools in its quest for service improvement. Some are derived from industry, others have been used in healthcare improvement programmes in the USA. All of our improvements need to be based firmly around the views of patients and carers and, to achieve this, the CHDC have pioneered a powerful tool called 'the discovery interview' which is used to elicit patients' and carers' experiences.

\section{DISCOVERY INTERVIEWS}

The CHDC, supported by the Critical Care Programme, has developed and tested this approach for improving services to patients. It is based on user narratives.

Discovery interviews are one of a number of possible approaches to involving patients and carers in improving care. They are particularly useful when clinical and other practice-based teams want to learn directly about patients needs and use this understanding to stimulate improvement ideas that they can test within their service.

One of the underpinning principals of continuous quality 
improvement work is that improving services should be about improving the way we meet the needs of those who are dependent on the NHS. It has been shown to be difficult to learn about patients' and carers' needs using traditional methods of asking questions about service provision, which are based on the professionals' expectations of what is important to users of services, rather than asking what is important to them. Professionals tend to believe that they know what patients want and often they are wrong. Explicit questions about needs can be difficult to answer. Yet unless we ask people what is important to them it is hard to see how the challenge of matching needs to service provision can be met.

The essence of the discovery interview process is listening to stories about the impact an illness or condition has had on patients' and carers' everyday lives, and linking what is heard with the professionals' knowledge and experience to improve the way in which needs are met.

Opportunities are created for providing information that is not constrained. The focus is shifted from enquiring about services to encouraging people to talk about themselves. People tell the story of their illness or condition in their own way, using a framework or 'spine' that guides them through the key stages of their experience. This provides information that local teams can use to identify needs and plan improvements in the ways that these can be met. The process is very much viewed as one of joint discovery and hence the interviews are called 'discovery interviews'.

An example of this is a story that described the walk from a car park to an outpatient clinic for a patient with heart failure, and how difficult and distressing this had been. Following the sharing of this story it was decided that bench seating should be placed at intervals along the way. This action has been much appreciated by patients and carers in the local area: a simple yet effective improvement used by many.

By September 2003, a training programme developed by the CHDC in 2002 had trained 250 CHD and critical care professionals in the use of the discovery interview. Over 300 had been carried out by the end of 2003. This training programme continues but plans are in place to run training locally from now.

Research Ethics Committees' approval both local and national is required and an application for this work to be carried out with CHD patients and carers has been submitted for the Morecambe Bay area. Local interviewers will be recruited by the project manager Sally Thornton with advice from trust managers, and training will be provided locally by the CHDC national team.

\section{USING THE DISCOVERY INTERVIEW TO LEARN FROM PATIENTS AND CARERS ABOUT BETTER WAYS TO MEET THEIR NEEDS}

One of the biggest challenges in healthcare today is finding viable ways to learn directly from patients and carers in the ongoing quest to improve NHS services. Confronting the idea of 'doctor knows best' is crucial for this challenge. Traditional approaches such as satisfaction questionnaires require value judgements from patients about their care, based on partial knowledge of their conuition and how services are run.

Discovery interviews have been developed to explore the impact of illness on patients' everyday lives during each stage of their journey through the healthcare system. Recruitment to the interview process is through clinical staff. Interviewers play no direct part in recruitment.

\section{TELLING THE STORY}

Specially-trained members of NHS staff carry out interviews in patients' and carers' homes. Using a tape recorder, interviewers make as few interruptions as possible to avoid influencing their subject's story.

The storyteller is guided by a framework, or 'spine' based on the key stages of their experience of illness. This triggers significant memories of how their health problems affect their lives.

\section{THE CHD SPINE}

thinking something was wrong

seeing someone in the NHS

having tests to find out what was wrong

being told what was wrong

receiving treatment

getting better

living with your condition

being followed up.

Their experience is naturally woven into their account and, even though it is not the primary focus of what they are saying, provides a useful insight into their underlying needs.

Clinical and other staff can use their clinical and professional knowledge and experience to interpret what they hear from patients and carers to create better ways of meeting their needs.

Stories generated by discovery interviews provide positive feedback to teams as well as drawing attention to areas that need to be improved. Improvement ideas usually take the form of small changes that are quickly implemented and tested to check whether they have improved care, or whether alternatives have to be found.

\section{BENEFITS OF USING THE SPINE FOR THE DISCOVERY INTERVIEW PROCESS}

- helps to cover the whole journey of the illness or condition under consideration

- triggers significant memories and allows the storytellers to use their own words

- allows the storytellers to remain in control of the interview

- provides rich information that can lead to better understanding, by offering context and perspective

- creates an immediacy that cannot be ignored, together with the desire to change things. 


\section{NUMBER OF DISCOVERY INTERVIEWS THAT NEED TO BE UNDERTAKEN}

Experience has shown that it is not the number of interviews conducted that is important, but the opportunity to hear detailed stories from a small number of service users from across the whole patient pathway. One story may provide many ideas for improvement work and those involved need to concentrate on the quality and number of improvements generated rather than the number of interviews completed.

\section{USING DISCOVERY INTERVIEWS TO IMPROVE CARE}

All interviews are recorded and then transcribed for sharing with clinical and multidisciplinary teams. During transcription, any references to people or places are removed to maintain confidentiality. References to services, however, such as $\mathrm{CCU}$ or A\&E, will be necessary to enable teams to identify where improvements should be made.

Using discovery interviews to prompt improvement activity has been most successful where clinical teams have been involved from the outset. It is also important for teams to plan arrangements for listening to stories and to agree that improvements will be required before the interviews are undertaken. It can take time for clinical teams to be comfortable with the process of listening to the stories and not feel unnecessarily criticised or defensive.

It is a key part of the discovery interview process that clinical teams should hear the story in the teller's own words. The evidence is that the listener hears and learns things that, otherwise, they would not. This strong patient or carer focus generates feelings in listeners that are a powerful motivator for improving the way things are done. Extracting themes out of context weakens the impact and may lead to defensive reaction. Teams tend to focus on the negatives and there is a tendency for the analyst to influence the list of themes identified.

Time must be planned for teams to hear the stories and plan action for improvement. It is crucial that time is allocated to allow serious discussion of the story and to make decisions about the next steps. It has become clear that the most effective way of listening to stories is to arrange for staff from different points on the journey to listen to them at the same time and then to act collectively on agreeing actions for testing changes that may lead to implementing improvements. Teams must be sure that changes will bring benefit to patients and carers and that there are no obvious risks.

\section{FEEDING BACK OUTCOMES}

It is critically important that storytellers receive feedback that will confirm that good use has been made of the time they spent telling their story. It must be explained, however, that feedback will not be given on specific incidents that may have been described, but rather will take the form of describing resulting improvements. So far feedback has been offered in the form of newsletters or by inviting people to join patients groups where improvements derived from listening to stories are discussed.

Making a firm commitment to feeding back serves as a healthy reminder that if they do not produce benefits discovery interviews are a waste of everybody's time.

Most of the experience of using discovery interviews to date is in CHD and critical care. Pilots are being planned for other areas of care, especially with vulnerable groups.

Stories generated by this type of interview provide positive feedback and draw attention to areas of service provision that need to be improved. Improvements usually take the form of small but significant changes that are quickly tested and implemented.

This technique is generating much interest in the wider NHS, principally because it clearly puts the patient at the centre of any improvements whilst encouraging patients and carers to tell us how it was from their point of view.

This is a very powerful tool for change and improvement and I am sure that as a tool it will continue to evolve whilst under the closest of scrutiny.

For more information on discovery interviews please visit www.modern.nhs.uk/chd or contact

tricia.myers@clha.nhs.co.uk

\section{REFERENCES}

1 Modern Standards and Service Models. National Service Framework for Coronary Heart Disease. DoH. March 2000

2 Mapping the Future. Coronary Heart Disease Collaborative Summary Strategic Plan. Modernisation Agency. August 2003

3 Improvement, Expansion and Reform: The Next Three Years. Priorities and Planning Framework 2003-2006. DOH 2002 